\title{
Effects of Angular Spread on the Performance in TD-SCDMA System with Smart Antenna
}

\author{
Shi Penglong, Ma Xiaoting, Chu Yaru, Ma Yizhong \\ Lanzhou University
}

\begin{abstract}
The received signal is correlated when applying the beamforming algorithm in the smart antennas system. Two main factors determine the un-correlation of the channel: the distance of antenna and the angular spread. In the paper a vector channel simulation model are presented based on geometrical environment and statistical data, then the vector channel correlation is analyzed. Subsequently, the beamforming algorithm which maximums the received desired power is adopted to analyze the effects of different angular spread on the downlink performance in TD-SCDMA system with smart antennas. Final the simulation result is presented.
\end{abstract}

Keywords-Smart antennas, TD-SCDMA, Angular spread

\section{I. .INTRODUCTION}

Nowadays, smart antennas is becoming more and more widely used in the wireless communication field because of its anti-jam and fixed position functions. On the uplink, we make full use of its space division character, change the receiver structure, then improve the performance of link level; on the downlink, we employ beamforming[3][4] to sending signal by use of weighted factor, make the sending signals to aim at the mobile station, accordingly reduce transmission power and Multi-Address Interference(MAI). Apply of various adaptive digital beamforming algorithms can adapt fast-growing business requirements, improve volume, covering and Quality of Services (QoS), therefore, it was recognized as one of the best solutions.

Many factors affect the performance of smart antennas, such as antenna pattern, antenna installed, the base station angle of arrival, angular spread and so on. In this paper, we major consider the angular spread factors caused by wireless channel, make analysis and simulation of smart antenna performance on the downlink.

Under circumstance that the take off angle and antenna array elements position have already known, the smaller the base station arrival angle the better the signal reached antenna array correspondingly, thus much able to reflect the efficiency of smart antennas. On the other hand it also means less space diversity gain, poorer anti-fading characteristics, just let the antenna pointing in the direction of useful users. Main factors that influence the base station angle of arrival are: 1.Shapes and sizes of scattering beam around the mobile station. Different scattering beam makes the angle of arrival distribution changed; 2.The distance from the scattering beam to base station. In the event of the same scattering beam shapes and sizes, the bigger the distance the smaller the spread of the arrival angle.

\section{SIGNAL AND CHANNEL MODEL}

In smart antenna system, firstly we need to model the vector channel. Vector channel can be divided into channel model based on geographical environment and channel model based on statistics data. For geography environment based channel model, we need to build its various channel environment parameters, such as number and distribution of scattering objects, distribution of base station and mobile station. Its computational complexity is very high, and need much more time to measure the systems performance; Statistics data-based channel model needs a lot of real data to analyze the characteristics of the channel. According to Gaussian Wide-Sense Stationary Uncorrelated Scattering Model (GWSSUS) [2], we described the relationship of mobile station, base station and scattering objects in wireless channel, show in Figure1.

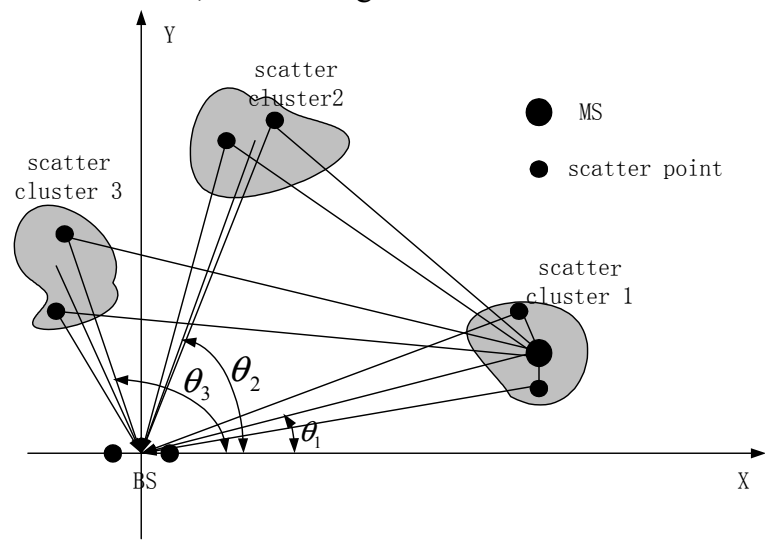

Figure 1. Schematic diagram of space vector channel

In this channel model, there are several large scattering clusters distribution around the mobile station, each scattering cluster is composed of numbers of scattering objects, and its scattering signal can be completely scattered. Mobile station on uplink sending omnidirectional signals, which go through the scattering clusters and final reach the base station, downlink keeps symmetrical with the uplink.

In this channel model, Multiple Input Multiple Output

(MIMO) vector channel formula [5] is:

$$
\mathbf{x}(t)=\sum_{l=0}^{L-1} \mathbf{A}_{l} \mathbf{a}\left(\theta_{l}\right) \alpha_{l}(t)_{s}\left(t-\tau_{l}\right)+\mathbf{n}(t)
$$

In formula (1), corresponds to the average angle of arrival of each scattering cluster, we assume that the angle from one scattering cluster to the base station obey Laplacian distribution, it can be expressed as follow: 


$$
\begin{array}{r}
f(\phi)=c \cdot \exp \left(-\left(\sqrt{2}\left|\phi-\theta_{l}\right|\right) / \sigma\right), \\
\phi-\theta_{l} \in(-\Delta \phi, \Delta \phi)
\end{array}
$$

Where $\mathrm{c}$ is a constant, $\sigma$ is the width of angular spread. In formula (1), $\mathbf{a}(\theta)$ is antenna array factor, which is determined by antenna array, considering the circle array [3], show in Figure2, $\alpha$ is incident angle, $D_{k}$ is the distance difference between the kth array element and the first array element.

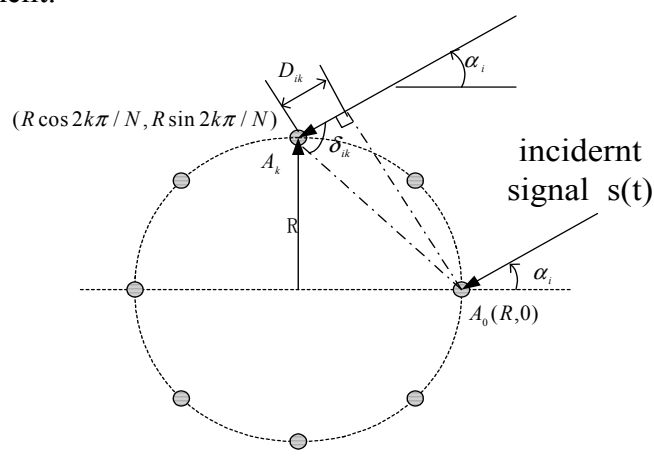

Figure 2. Schematic diagram of circular array antenna Antenna array factor can be written as:

$$
\mathbf{a}(\theta)=\left[\begin{array}{llll}
1 & e^{-j \varphi_{2}} & \ldots & e^{-j \varphi_{N-1}}
\end{array}\right]^{T}
$$

In which,

$$
\begin{aligned}
& \varphi_{k}=2 \pi \frac{D_{k}}{\lambda} \\
& D_{k}=\cos \delta_{k} * R \sqrt{2(1-\cos 2 k \pi / N)} \\
& \delta_{k}=\alpha+\left(\frac{1}{2}-\frac{k}{N}\right) \pi
\end{aligned}
$$

If the scattering cluster radius is smaller, then we can assume that delay of one scattering cluster to the base station cannot be distinguished, $\tau_{l}$ is the average delay of each scattering cluster; $\mathrm{L}$ is number of scattering cluster; $\alpha_{l}$ is fading characteristics of each scattering cluster, so that range meets the Rayleigh distribution, phase meets the uniform distribution. Considering the circumstance that all power of transmitting signal which from the mobile station were scattered to base station by scattering objects, we assume that Doppler spectrum was classical U type, so Doppler spectrum density can be expressed as the following form[1],

$$
S(f)=\frac{k}{\sqrt{f_{m}^{2}-\left(f-f_{c}\right)^{2}}},\left|f-f_{c}\right|<f_{m}
$$

In which, $\mathrm{k}$ is the normalization factor, $f_{m}$ is the maximum Doppler shift, $f_{c}$ is the centre frequency.

Angle and angular spread influence the relevance of the antenna array. In formula (1), $\mathbf{A}_{l}$ means the relevance of the $l^{\text {th }}$ scattering cluster of the antenna array side caused by angle and angular spread, we use correlation matrix to represent this correlation. Correlation matrix is a matrix constituted by correlation coefficient of each two antennas, which is defined [6] as follows,

$$
\rho=\left\langle s_{a}, \quad s_{b}\right\rangle=\frac{E\left(s_{a} s_{b}^{*}\right)-E\left(s_{a}\right) E\left(s_{b}^{*}\right)}{\sqrt{\operatorname{Var}\left(s_{a}\right)} * \sqrt{\operatorname{Var}\left(s_{b}\right)}}
$$

In formula (8), $s_{a}, s_{b}$ are signal of the antenna array element $\mathrm{a}$ and $\mathrm{b}$, which are expressed as,

$$
\begin{gathered}
s_{a}(t)=\frac{1}{2 \Delta \phi} \int_{-\Delta \phi}^{\Delta \phi} s(t) * \exp \left(-j 2 \pi l \sin \left(\phi+\theta_{l}\right) / \lambda\right) d \phi \\
s_{b}(t)=\frac{1}{2 \Delta \phi} \int_{-\Delta \phi}^{\Delta \phi} s(t) * \exp \left(-j 2 \pi(d+l) \sin \left(\phi+\theta_{l}\right) / \lambda\right) d \phi
\end{gathered}
$$

The symbols are defined as in formula (2), calculate $\rho_{a b}$ of every two array elements, and construct correlation matrix as follow,

$$
\mathbf{A}_{l}=\left(\begin{array}{ccc}
\rho_{11} & \cdots & \rho_{1 N} \\
\vdots & \ddots & \vdots \\
\rho_{N 1} & \cdots & \rho_{N N}
\end{array}\right)
$$

In which, $\rho_{a b}=\rho_{b a}^{*}$.

Through the above analysis we can see that, comparing with traditional channel characters, vector channel imports two parameters: antenna array element and correlation matrix, so simulation of vector channel is much more complicated than that of tradition channel. While do analysis and simulation to the downlink, downlink channel using the MISO channel, that is multiple antennas single antenna reception. In order to get the maximum received power of useful user reaching the mobile station, we adopt maximum received power algorithm [7] [8] to obtain a set of weighted factors, make the follow formula,

$$
\mathbf{R}^{(k)}=\max _{a v g \mathbf{W}^{(k)}} \mathbf{W}^{(k)^{H}} \mathbf{R}_{n}^{(k)} \mathbf{W}^{(k)}
$$

satisfies the normalized at the same time, that is,

$$
\begin{aligned}
& \left\|\mathbf{W}^{(k)}\right\|=1 \\
& \text { In formula (12), } \mathbf{R}_{n}^{(k)}=\mathbf{h}^{(k)^{*}} \mathbf{h}^{(k)^{T}}
\end{aligned}
$$

In which, $\mathbf{h}^{(k)}$ is the $\mathrm{k}$ users' downlink channel shock response.

As TD-SCDMA system is the TDD mode, and its subframe interval is $5 \mathrm{~ms}$, so when mobile station running at low speed, what we only need to do is estimate information of the uplink channel, then approximated the downlink channel shock response by using it.

\section{SIMULATION PARAMETERS AND SIMULATION}

\section{RESULTS}

In downlink wave cluster beamforming simulation platform, according to uplink channel information, each user gets a set of downlink weighting factors, weighted by weighting factor then passes through the antenna, after MISO channel, and in the mobile station use one antenna to 
receive it. Some important parameters are show in follow table.

Table I. Basic simulation parameters

\begin{tabular}{|l|l|}
\hline Parameters & \multicolumn{1}{|c|}{ Parameter values } \\
\hline Business Rate & $12.2 \mathrm{~kb}$ \\
\hline Chip Rate & $1.28 \mathrm{Mcps}$ \\
\hline Modulation Mode & QPSK \\
\hline Spreading Factor & 16 \\
\hline Total User & 5 \\
\hline Carrier Frequency Array Elements & $8 \mathrm{GHz}$ \\
\hline $\begin{array}{c}\text { Antenna Aumber } \\
\text { Nurcular Array Radius }\end{array}$ & $\lambda / 2$ \\
\hline $\begin{array}{c}\text { Velocity of the Mobile station } \\
\text { (v) }\end{array}$ & $3 \mathrm{~km} / \mathrm{h}$ \\
\hline Angular Spread & $1^{\circ}, 5^{\circ}, 10^{\circ}$ \\
\hline For Rayleigh multipath parameters of simulation
\end{tabular}
channel, we choose 3GDP to measure CASE1 parameter in the channel, so that there are two scattering clusters, their fading are $0 \mathrm{~dB}, 10 \mathrm{~dB}$, meanwhile, each scattering cluster corresponds to a different angle, and assuming that scattering clusters are evenly distributed around the base station.

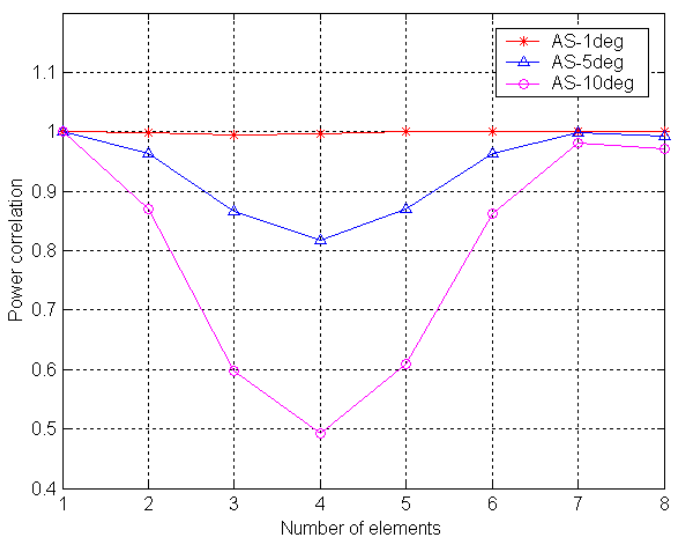

Figure 3. Circular array antenna correlation diagram

Figure 3 is circular array antenna power correlation diagram, angular spread of the three curves are $1^{\circ}, 5^{\circ}, 10^{\circ}$.From it we can see that, the correlation among antenna array elements continuous decline when the angular spread increases.

In Figure 4, at the same RawBER value, different angular spreads have different performance. It can be seen, when the angular spread is smaller performance is poor, when angular spread is bigger there is a certain improvement in performance. We also can see that, when RawBER $=10^{-2}, 10^{\circ}$ angular spread have nearly $3 \mathrm{~dB}$ performance improvement than $1^{\circ}$ angular spread. This is because when the angular spread changes within a certain range, it has certain effect to performance of the system, so in different environments, the performance of smart antennas exists big differences.

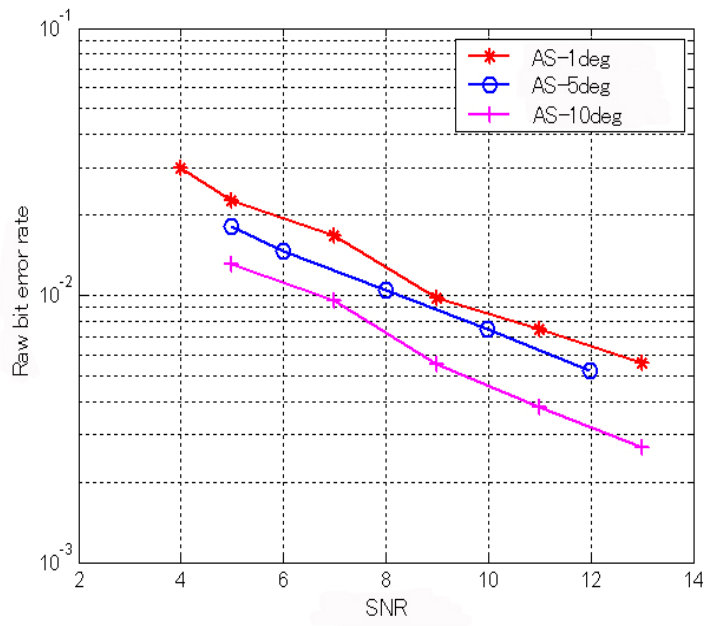

Figure 4. The relationship between transmission power and RawBER

\section{SUMMARIES}

In wireless channel, changes in scattering cluster size can lead to changing of angular spread; In smart antenna system, received signals are related, and all antenna array elements have same fading at the same time; But when the angular spread is getting bigger, relation of received signals is getting smaller, thus obtain spatial diversity gain and making the TD-SCDMA smart antenna system performance is improved.

\section{REFERENCES}

[1] W. C. Jakes. Microwave Mobile Communication. New York: Wiley 1974

[2] Joseph C. Liberti; Jr and T. S. Rapport. Smart antenna for wireless communications: IS-95 and third generation CDMA applications. Prentice Hall PTR, 1999

[3] John Litva and Titus Kwok-Yeung Lo. Digital beamforming in wireless communications. Artech House, 1996

[4] L. C. Godara. Application of antenna arrays to mobile communications, Part 2: beamforming and direction-of-arrival considerations. Proceedings of the IEEE, Vol.85, NO.8, AUG 1997

[5] Laurent Schumacher; Jean Philippe Kermoal. Multi-element transmit and receive antenna - MIMO channel characterization. METRA, 2000

[6] William C.Y. LEE. Effects on correlation between two mobile radio base-station antenna. IEEE transaction on communication, Vol.Com21, NO.11, Nov., 1973

[7] 3GPP R1 99623. Smart Antenna Technology. IST-1999-117291 METRA D3.2 Review and Selection of Relevant Algorithms. 